Preprint typeset in JHEP style. - HYPER VERSION

ATL-PHYS-PUB-2005-023

\title{
Measuring slepton spin at the LHC
}

\author{
A.J. Barr \\ Department of Physics 83 Astronomy, University College London, Gower Street, \\ LONDON, WC1E 6BT
}

\begin{abstract}
A new method is presented for measuring the spin of selectrons and smuons at the Large Hadron Collider (LHC), using an angular variable which is sensitive to the polar angle in direct slepton pair production. This variable, $\cos \theta_{l l}^{*}$, is invariant under boosts along the beam axis, so it can be used at the LHC despite the fact that the longitudinal boost of the centre-of-mass frame cannot be determined. Monte Carlo simulations demonstrate that, using this method, the LHC can distinguish between the supersymmetric production angular distribution and phase space, or between supersymmetry and the production angular distribution of universal extra dimensions. An integrated luminosity of about 100 to $300 \mathrm{fb}^{-1}$ provides sufficient statistics to measure the slepton spin for points which had left-handed slepton masses in the range 202 to $338 \mathrm{GeV}$, and right-handed sleptons in the range 143 to $252 \mathrm{GeV}$. Good sensitivity was found in the 'bulk' and 'stau co-annihilation' regions of the cMSSM favoured by cosmological relic density measurements. Various systematic uncertainties are investigated, and some methods for reducing them are discussed.
\end{abstract}

KEYWORDs: Hadronic Collider, LHC, Supersymmetry, Spin, ATLAS, Universal Extra Dimensions. 


\section{Contents}

1. Introduction 1

2. Angular distributions, and $\cos \theta_{l l}^{*}$

3. Monte Carlo simulation of test points 5

3.1 Event selection 6

3.2 Results 9

4. Systematic uncertainties 14

4.1 Lepton efficiency, acceptance and charge identification 14

4.2 Standard Model background determination 15

4.3 SUSY backgrounds 15

4.4 SUSY spectrum 16

4.5 Migrations at cut boundaries 17

4.6 Migrations between bins 18

4.7 Summary of systemic uncertainties 18

5. Conclusions 18

\section{Introduction}

One of the main goals of the LHC is to search for new physics, beyond the Standard Model (SM). In most cases, if new particles exist at the TeV-scale, then the LHC experiments will be able to discover their existence. But discovery is only the first step - to distinguish between models, the properties of the new particles also need to be experimentally determined.

Probably the leading contender for new physics at the TeV scale is supersymmetry (SUSY). The minimal supersymmetric extension to the SM predicts that each SM particle should have a partner with spin differing by $\frac{1}{2}$. If R-parity is conserved, then the supersymmetric partners must be pair-produced and the lightest supersymmetric particle (LSP), typically the $\tilde{\chi}_{1}^{0}$, is stable. The typical LHC signature is therefore a pair of cascade decays which produce jets and leptons, as well as missing energy from the (assumed invisible) lightest supersymmetric particle.

There are, however many other competing models for new physics at the TeV scale besides supersymmetry. Indeed it has recently been appreciated that models 
with Universal Extra Dimensions (UED) and Kaluza-Klein (KK) parity [1] could have very similar collider phenomenology to supersymmetric model [2].

The minimal version of UED predicts that for each SM particle there should be a tower of Kaluza-Klein (KK) excitations. KK parity means that particles with odd KK-number, such as the first excited state of any SM particle can only be produced in pairs. It also ensures that the lightest KK particle must be stable, in the same way as R-parity does for supersymmetry. Distinguishing between SUSY and UED could therefore be a difficult problem, since both models predict $\mathrm{TeV}$-scale pair-produced particles which decay through cascades with Standard Model couplings, with the eventual production of a pair of invisible daughters (LSP or LKP).

While other measurements might be indicative [3,4], the property which will a give conclusive answer as to whether we are observing SUSY or UED is the spin of the excited particles.

Recently some progress has been made towards spin-determination of supersymmetric particles at the LHC. The method, suggested in [5] and investigated in [3, 6,7], involved measurement of the lepton charge asymmetry in $\ell q$ invariant mass distributions in the cascade decay,

$$
\tilde{q}_{L} \rightarrow \tilde{\chi}_{2}^{0} q_{L} \rightarrow \tilde{l}_{R}^{ \pm} l^{\mp} q_{L} .
$$

That measurement was shown to have sensitivity to the spin of the $\tilde{\chi}_{2}^{0}$. While it was comforting to see that the LHC can have sensitivity to sparticle spins, the caveat is that in some parts of parameter space, the decay chain eq. 1.1 is kinematically forbidden or has a small branching ratio. This makes it important to investigate other channels and other particles for which the LHC experiments could measure spin.

In this paper we present a new method for measuring slepton spin at the LHC. The paper is organised as follows. In section 2 we introduce an angular variable $\cos \theta_{l l}^{*}$, and show that it is sensitive to the production polar angle in slepton pair production. Our supersymmetric test points, Monte Carlo event generator and detector simulation are described in section 3. In section 3.1 we identify an event selection and demonstrate that it can cleanly isolate the signal process. Results showing the experimentally-measurable angular distributions and luminosity requirements are shown in section 3.2. In section 0 we discuss the main systematic uncertainties and some methods for reducing them. Our conclusions are presented in section 5 .

\section{Angular distributions, and $\cos \theta_{l l}^{*}$}

In this paper we investigate the supersymmetric process,

$$
q \bar{q} \rightarrow Z^{0} / \gamma \rightarrow \tilde{\ell}^{+} \tilde{\ell}^{-} \rightarrow \tilde{\chi}_{1}^{0} \ell^{+} \tilde{\chi}_{1}^{0} \ell^{-},
$$


where throughout this paper $\ell$ is understood to mean electron or muon only. Since sleptons are scalars, the angluar distribution for Drell-Yan slepton pair production is

$$
\left(\frac{d \sigma}{d \cos \theta^{*}}\right)_{\mathrm{SUSY}} \propto 1-\cos ^{2} \theta^{*}
$$

where $\theta^{*}$ is the angle between the incoming quark in one of the protons and the produced slepton. Slepton pair production via gauge boson fusion [8] is not considered here, but it would become important for sleptons with masses greater than about $300-400 \mathrm{GeV}$. For comparison we use a pure phase space distribution,

$$
\left(\frac{d \sigma}{d \cos \theta^{*}}\right)_{\mathrm{PS}} \propto \text { constant . }
$$

The phase space distribution does not correspond to any physical model, but does provide a convenient benchmark against which to compare the SUSY distribution.

We also compare against the UED equivalent of eq. 2.1,

$$
q \bar{q} \rightarrow Z^{0} / \gamma \rightarrow \ell_{1}^{+} \ell_{1}^{-} \rightarrow \gamma_{1} \ell^{+} \gamma_{1} \ell^{-}
$$

which has the characteristic distribution for spin- $\frac{1}{2}$ KK leptons:

$$
\left(\frac{d \sigma}{d \cos \theta^{*}}\right)_{\mathrm{UED}} \propto 1+\left(\frac{E_{\ell_{1}}^{2}-M_{\ell_{1}}^{2}}{E_{\ell_{1}}^{2}+M_{\ell_{1}}^{2}}\right) \cos ^{2} \theta^{*},
$$

where $E_{\ell_{1}}$ and $M_{\ell_{1}}$ are the energy and mass

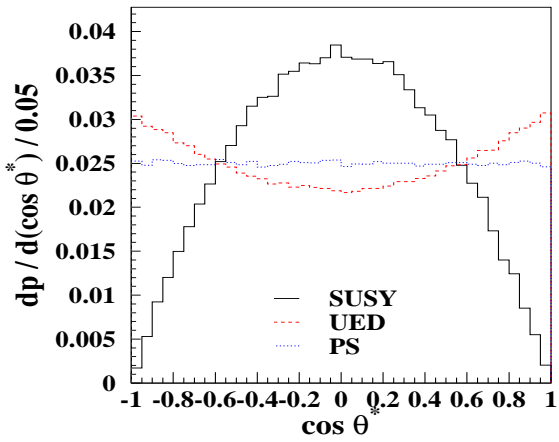
respectively of the KK leptons in the centerof-mass frame. The three different pro- Figure 1: Production angular distribuduction angular distributions are shown graptitions, $\frac{d p}{d \cos \theta^{*}}$, for scalar sleptons (SUSY), ically in fig. 1.

The different angular distributions provide a mechanism for determining the heavy particle spin. Excited leptons (selptons or spin- $\frac{1}{2} \mathrm{KK}$ leptons UED and pure phase space (PS). The mass spectrum for the UED distribution is that of SUSY point S5 (see section [).

KK-leptons) which are produced significantly above threshold will have decays which are boosted in the lab frame. This means that a pair of leptons from slepton decays (eq. 2.2) should be on average less widely separated in polar angle than the pair from phase space (eq. 2.3) or KK-lepton pair production (eq. 2.5).

It has already been suggested $[9,10]$ that the final state lepton angular distributions could be used at a future high-energy $e^{+} e^{-}$linear collider to distinguish between UED and SUSY models. With a proton-proton collider such as the LHC, it is not possible to measure the lepton angluar distributions in the parton-parton center-of-mass frame - the initial z-momenta of the incoming partons are not known, 

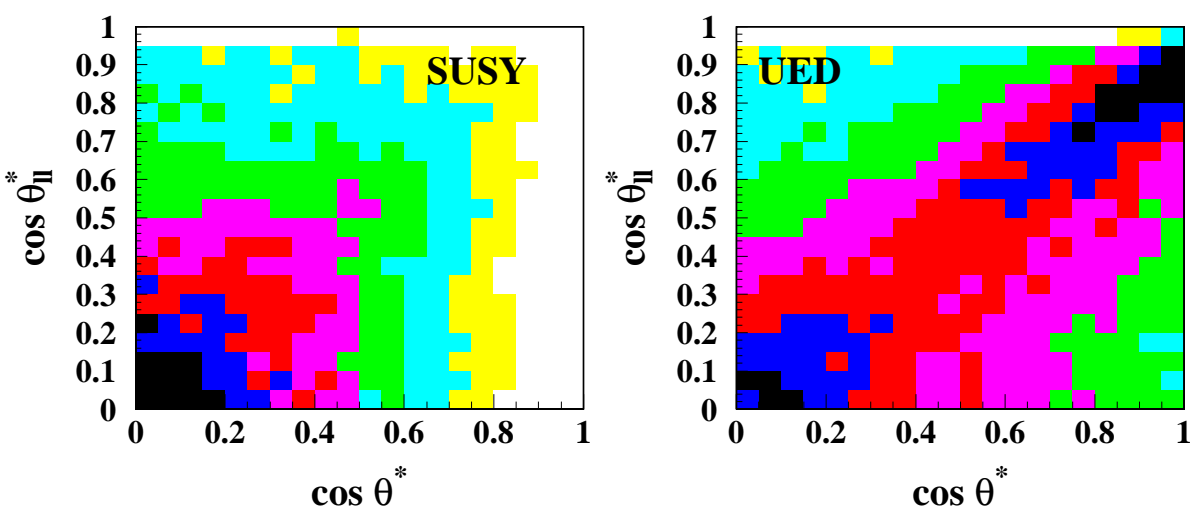

Figure 2: 2-dimensional plots showing the correlation between our dilepton angular variable, $\cos \theta_{l l}^{*}$, (y-axes) and the cosine of the production angle of the parent sleptons (a) or KK-leptons (b) in the center of mass frame (x-axes). Darker regions correspond to larger numbers of events, the normalisation being arbitrary. The mass spectrum is that of SUSY point S5.

and because invisible particles are produced, the center of mass frame of the partonparton interaction cannot be recovered from the final state.

To make a spin measurement at a hadron collider, we propose a variable which is a function only of the pseudorapidity difference between the final state leptons, $\Delta \eta_{\ell^{+} \ell^{-}}$. The advantage of differences in rapidity is that they are independant of the longitudinal boost. The leptons are highly relativistic, so we can use their pseudorapidities as a very good approximation to their true rapidities. By using a function only of $\Delta \eta_{\ell^{+} \ell^{-}}$, we no longer have to face the problem of determining the centerof-mass frame along the beam direction. The inter-lepton pseudorapdity difference, $\Delta \eta_{\ell^{+} \ell^{-}}$, is also sensitive to the slepton production angle. The reasons are the same reasons as for the lepton angular distributions - the leptons 'inherit' some knowledge of the rapidity of their slepton or KK-lepton parents. Lepton pairs from slepton pair decay will therefore be on average less separated in pseudorapidty than those coming from particles produced according to the corresponding phase-space or Kaluza Klein production angular distributions.

To allow a more direct comparison with the production distributions, rather than using $\Delta \eta_{\ell^{+} \ell^{-}}$directly, we propose the angular variable

$$
\cos \theta_{l l}^{*} \equiv \cos \left(2 \tan ^{-1} \exp \left(\Delta \eta_{\ell^{+} \ell^{-}} / 2\right)\right)=\tanh \left(\Delta \eta_{\ell^{+} \ell^{-}} / 2\right)
$$

This variable, like $\Delta \eta_{\ell^{+} \ell^{-}}$, has the benefit of being longitudinally boost invariant, but also has a simpler geometrical interpretation: $\cos \theta_{l l}^{*}$ is the cosine of the polar 
angle between each lepton and the beam axis in the longitudinally boosted frame in which the (pseudo-)rapidities of the leptons are equal and opposite.

As can be seen from fig. 2, $\cos \theta_{l l}^{*}$ is indeed well correlated with the slepton or KK-lepton production angle, $\cos \theta^{*}$. The experimental observable $\cos \theta_{l l}^{*}$ is on average smaller for SUSY (fig. 2a) than for UED (fig. 2/b), meaning that $\cos \theta_{l l}^{*}$ can be usefully employed as a spin-sensitive discriminant in slepton/KK-lepton pair production at hadron colliders.

\section{Monte Carlo simulation of test points}

We chose as a primary test point the LHC point 5 [11], (S5), with supersymmetric mass spectrum and branching ratios calculated with ISAJET-7.64. Further simulations were performed with the Snowmass points SPS1a, SPS1b, SPS2, SPS3, SPS4, SPS5, and SPS6 [12] with spectra and branching ratios calculated using ISAJET-7.58, following the Snowmass standard.

Some important masses as well as the signal cross-section are given in table 1 . It is immediately clear that SPS2, which is in the 'focus point' ${ }^{1}$ region, and has very heavy sleptons, will not provide a measurable signal at the LHC. Of the other points, we note that SPS4 has slepton masses near the edge of our expected sensitivity for direct production [14]. The non-universal point SPS6 has a smaller-than-usual mass difference between the sleptons and the LSP, and so will produce rather soft leptons in its decays.

The Monte Carlo event generator used was HERWIG-6.507 [15-17], with the parton distributions of MRST [18] (average of central and higher gluon). Since a complete

\begin{tabular}{|c|c|c|c|c|c|}
\hline \multicolumn{6}{|l|}{$\begin{array}{l}{ }^{1} \text { For a discus } \\
\text { example, [13]. }\end{array}$} \\
\hline & Point & $\tilde{\chi}_{1}^{0}$ & $\tilde{e}_{R}$ & $\overline{\tilde{e}_{L}}$ & $\sigma\left(p p \rightarrow \ell^{+} \ell^{-}\right)$ \\
\hline & S5 & 116 & 153 & 229 & $65 \mathrm{fb}$ \\
\hline & SPS1a & 96 & 143 & 202 & $88 \mathrm{fb}$ \\
\hline & SPS1b & 160 & 252 & 338 & $12 \mathrm{fb}$ \\
\hline & SPS2 & 80 & 1452 & 1456 & $0.003 \mathrm{fb}$ \\
\hline & SPS3 & 161 & 178 & 287 & $34 \mathrm{fb}$ \\
\hline & SPS4 & 119 & 417 & 448 & $2.5 \mathrm{fb}$ \\
\hline & SPS5 & 120 & 191 & 256 & $48 \mathrm{fb}$ \\
\hline & SPS6 & 189 & 237 & 265 & $22 \mathrm{fb}$ \\
\hline
\end{tabular}

Table 1: Masses of selected particles (in $\mathrm{GeV}$ ) for the model points investigated, and the sum of the leading order cross-sections for direct slepton $\left(e^{+} e^{-}\right.$and $\left.\mu^{+} \mu^{-}\right)$pair production. 
UED Monte Carlo event generator programme was not available, HERWIG was modified to generate slepton pairs with the other production angular distributions, eq. 2.3 and 2.5. The slepton angular distribution (eq. 2.2) is of course already implemented in HERWIG [16]. Angular distributions for UED processes other than for $\ell_{1}^{+} \ell_{1}^{-}$production in eq. 2.4 were not modified. In general, this should not significantly affect the results presented, since cuts were later applied (section 3.1) which reduced the residual supersymmetric background to a small fraction of the signal.

Unlike the case of slepton decay, the extent to which the spin structure of the $\ell_{1} \rightarrow \gamma_{1} \ell$ vertex is important will depend on the details (such as the KK Weinberg angle and left-right $\ell_{1}$ mixings) of the UED model. We expect that others with an interest in particular UED models will investigate in more detail the performance of this method for those models. For this paper, we merely note that the KK-leptons are only weakly polarised (as can be seen from the similarity between the UED and PS distributions in fig. 1), and so in our simple approximation to UED we ignore spin correlations in the $\ell_{1}$ decays.

Samples of inclusive supersymmetric particle production were generated corresponding to integrated luminosities from 25 to $500 \mathrm{fb}^{-1}$ for each of the test points. Also, $500 \mathrm{fb}^{-1}$ samples were generated for the major backgrounds, $W^{ \pm} W^{\mp}, Z^{0} Z^{0}$, $W^{ \pm} Z^{0}$, and $t \bar{t}$. To understand the 'theoretical' distributions, very large luminosity $\left(10 \mathrm{ab}^{-1}\right)$, samples were generated of the signal process for each of the three production angular distributions (eqs. 2.2, 2.3 and 2.5), and for each test point. In total about $8 \times 10^{8}$ events were generated.

All of the events generated were passed through the ATLAS fast detector simulation programme ATLFAST-2.50 [19], which contains a parameterisation of the ATLAS detector response for leptons, jets and missing energy. Jets were defined by having $10 \mathrm{GeV}$ of transverse energy in a cone of radius 0.4 . The pseudorapidity coverage for jets was $\left|\eta_{j}\right|<5$, while the lepton $(\ell \in e, \mu)$ pseudorapidity coverage was $\left|\eta_{\ell}\right|<2.5$. We assume a $b$-jet vertex tagging efficiency of $60 \%$, a $c$-jet vertex tagging rate of $10 \%$ and light-quark jet mistagging rate of $1 \%$ [11].

\subsection{Event selection}

The final state of interest consists of two opposite-sign, same family (OSSF) leptons, either electrons or muons, together with two invisible particles. Thus the initial selection is a requirement that exactly two OSSF leptons are detected, and that there must be missing transverse momentum, $\not p_{T}>100 \mathrm{GeV}$. The lepton with the larger transverse momentum must satisfy $p_{T}\left(\ell^{(1)}\right)>40 \mathrm{GeV}$, while that with the smaller $p_{T}$ must have $p_{T}\left(\ell^{(2)}\right)>30 \mathrm{GeV}$. This lepton momentum selection also ensures that these events satisfy the ATLAS trigger requrements.

The main Standard Model backgrounds are expected to come from the final state $\ell^{+} \ell^{-} \nu \bar{\nu}$, from $W^{ \pm} W^{\mp}$ or $Z^{0} Z^{0}$ production. Since $W^{ \pm} Z^{0}$ and $t \bar{t}$ might also be significant, they were also generated. 
The $Z^{0} Z^{0}$ backgrounds is largely removed by excluding events with dilepton invariant mass $m_{\ell \ell}<150 \mathrm{GeV}$.

The $W^{ \pm} W^{\mp}$ pair background can be substantially reduced by making a cut on a different kinematic variable, known as $m_{T 2}$. This variable, described in [20,21], is similar to the transverse mass, $m_{T}$, but is useful in events in which a pair of same-mass particles decays semi-invisibly. It is defined by:

$$
m_{T 2}^{2}\left(m_{\nu}\right) \equiv \min _{\boldsymbol{\phi}_{T}^{(1)}+\boldsymbol{\phi}_{T}^{(2)}=\mathbf{p}_{T}}\left[\max \left\{m_{T}^{2}\left(\mathbf{p}_{T}^{\ell^{(1)}}, \boldsymbol{\phi}_{T}^{(1)} ; m_{\nu}\right), m_{T}^{2}\left(\mathbf{p}_{T}^{\ell^{(2)}}, \boldsymbol{\phi}_{T}^{(2)} ; m_{\nu}\right)\right\}\right]
$$

where,

$$
\begin{aligned}
& m_{T}^{2}\left(\mathbf{p}_{T}^{\ell}, \mathbf{p}_{T}^{\nu} ; m_{\nu}\right) \equiv m_{\ell^{+}}^{2}+m_{\nu}^{2}+2\left(E_{T}^{\ell} E_{T}^{\nu}-\mathbf{p}_{T}^{\ell} \cdot \mathbf{p}_{T}^{\nu}\right), \\
& E_{T}^{\ell}=\sqrt{\left(\mathbf{p}_{T}^{\ell}\right)^{2}+m_{\ell}^{2}} \quad \text { and } \quad E_{T}^{\nu}=\sqrt{\left(\mathbf{p}_{T}^{\nu}\right)^{2}+m_{\nu}^{2}} .
\end{aligned}
$$

and $\mathbf{p}_{T}^{\ell}$ and $\mathbf{p}_{T}^{\nu}$, indicate the lepton and neutrino transverse momenta respectively ${ }^{2}$. $m_{T 2}$ has the property by construction that for events in which the lepton pair originates from on-mass-shell $W^{ \pm} W^{\mp}$ pairs,

$$
m_{T 2}\left(\mathbf{p}_{T}^{\ell^{+}}, \mathbf{p}_{T}^{\ell^{-}} ; m_{\nu}\right)<m_{W} .
$$

We therefore exclude from our analysis any event for which $m_{T 2}\left(\mathbf{p}_{T}^{\ell^{+}}, \mathbf{p}_{T}^{\ell^{-}} ; 0\right)<$ $100 \mathrm{GeV}$, removing most of the $W^{ \pm} W^{\mp}$ background.

To reduce the background from $t \bar{t}$ production and from cascade decays of heavier KK or SUSY particles, the following additional selection was applied:

- no jet with $\mathbf{p}_{T}>100 \mathrm{GeV}$

- no jet with a vertex tag (no ' $b$-jets')

- transverse recoil, $\left|\boldsymbol{p}_{T}+\mathbf{p}_{T}\left(\ell^{+}\right)+\mathbf{p}_{T}\left(\ell^{-}\right)\right|<100 \mathrm{GeV}$,

where the jet and vertex tagging algorithm parameters are as described previously.

Another potential background comes from single $W^{ \pm}$production in association with a jet or photon which fakes a lepton in the detector. The evaluation of such backgrounds would require a complete detector description, beyond the scope of this paper, and so they have not been included in this analyis. In fact we expect that although they have a much higher cross-section than gauge boson pair production, they should not pose a serious threat. The expected rate of jets faking electrons is rather small - of the order $10^{-4}-10^{-5}$ [11]. What is more, these types of event should also be efficiently removed by the cuts. Fake events where the $W^{ \pm}$is close to its mass shell and in addition either:

\footnotetext{
${ }^{2}$ The neutrino mass is not important in this analysis, but is included in eqs. 3.13 .3 for completeness.
} 
- there is no significant missing transverse momentum $\left(\boldsymbol{p}_{T}\right)$ except that caused by the neutrino from the $W^{ \pm}$decay or

- any additional contribution to $\boldsymbol{p}_{T}$ is collinear with the 'fake' lepton, as could be expected from a poor energy measurement of that fake,

will fail the $m_{T 2}$ cut. The reason can be understood when one considers the quantity being minimized in eq. 3.1 with a particular pair of value for the 2-vectors $\boldsymbol{\phi}_{T}^{(1)}$ and $\phi_{T}^{(2)}$ which represent a possible transverse missing energy spitting. The interesting configuration is one in which $\phi_{T}^{(1)}$ is equal to the true neutrino transvers momentum, and $\boldsymbol{\phi}_{T}^{(2)}$ is parallel to the fake lepton. Under the conditions above, this configuration will satisfy the constraint $\mathbf{q}_{T}^{(1)}+\mathbf{q}_{T}^{(2)}=\boldsymbol{p}_{T}$. The first transeverse mass, $m_{T}^{(1)}$, containing the neutrino and lepton from the $W^{ \pm}$decay, must be less than $m_{W}$, while the other, $m_{T}^{(2)}$, containing the fake lepton, will be close to zero. Since such a configuration exists, it places an upper limit on $m_{T 2}$ of $m_{W}$.

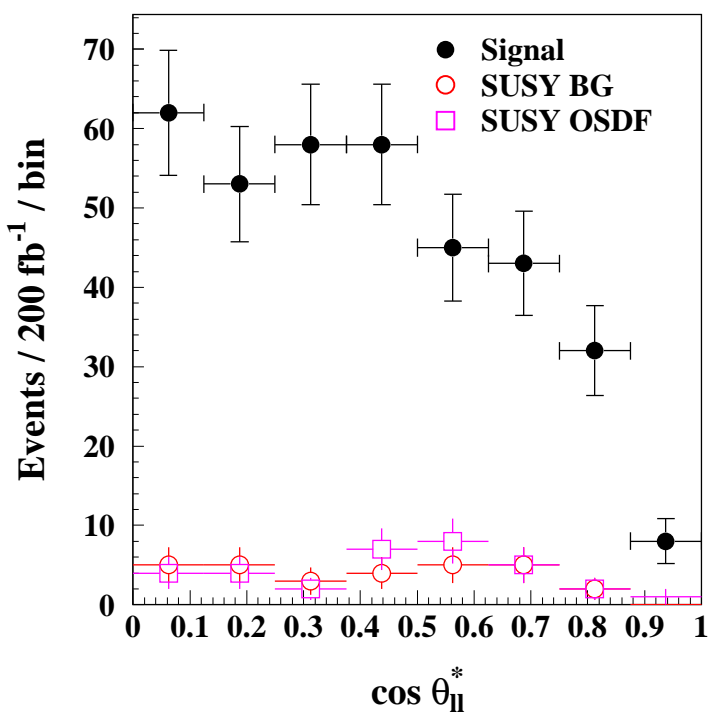

Figure 3: Distribution of $\cos \theta_{l l}^{*}$ for the S5 SUSY sample after event selection. The dark cicles represents the SUSY slepton pair signal (eq. 2.1). The open cicles indicate the SUSY background. The open squares show the different flavour (OSDF) estimate of the SUSY background, which is explained in section 4.4. In this and in all subsequent plots, the electron-pair and muon-pair distributions have been combined.

The cuts are somewhat similar to those used for studies of slepton discovery potential, or mass measurements from Drell-Yan pair production at the LHC [14, 22-26]. One notable difference is that, unlike most previous studies, we make no 


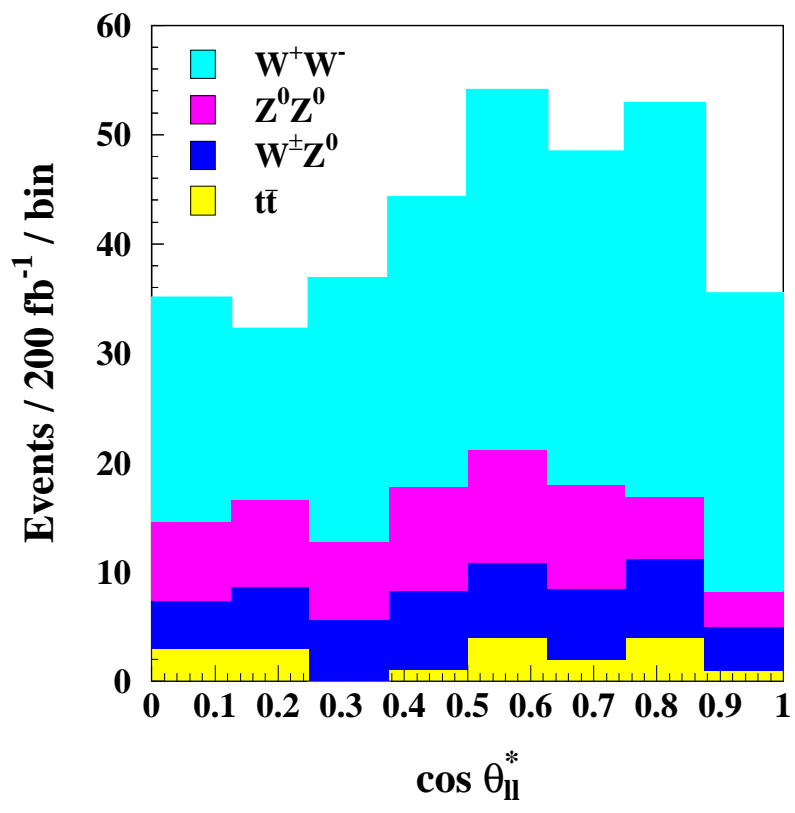

Figure 4: The Standard Model backgrounds after the cuts have been applied, as a function of $\cos \theta_{l l}^{*}$.

azimuthal angle $(\phi)$ requirements on the leptons or $\boldsymbol{p}_{T}$ vector, since this seem to be unnecessary when an $m_{T 2}$ cut is used to reduce the $W^{ \pm} W^{\mp}$ background.

Fig. 3 shows that after the cuts have been applied, the SUSY background is very much smaller than the signal. Similarly fig. A demonstrates that the Standard Model backgrounds do not overwhelm the SUSY signal. However the SM background is not insignificant, and so it will have to be accurately determined if a slepton spin measurement based on $\cos \theta_{l l}^{*}$ is to be achieved. The degree to which these backgrounds can be understood is investigated further in sections 4.2 and 4.3 .

\subsection{Results}

In this section we disuss the $\cos \theta_{l l}^{*}$ distributions which would be obtained assuming that the supersymmetric and Standard Model backgrounds can be accurately determined. We then go on to examine the extent to which these assumptions can be justified in section 4 .

In fig. 5 we plot the fraction of events as a function of $\cos \theta_{l l}^{*}$ for $200 \mathrm{fb}^{-1}$ of integrated luminosity. As has already been shown in figs. 1 and 5, for point S5 the statistical uncertainty on the SUSY signal and the SM background are comparable, while the SUSY background is small. It is clear that the (SUSY) "data" sample is much better matched to the slepton angular distribution than to either the phase- 


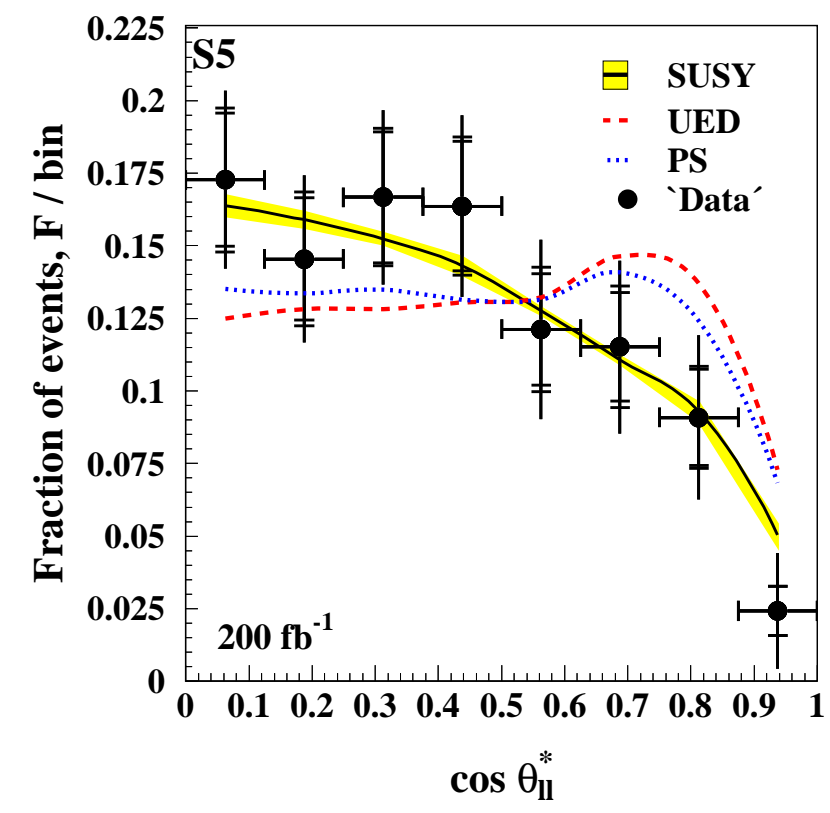

Figure 5: The points show the $\cos \theta_{l l}^{*}$ distribution for the S5 signal sample $\left(\tilde{\ell}^{+} \tilde{\ell}^{-} \rightarrow\right.$ $\tilde{\chi}_{1}^{0} \ell^{+} \tilde{\chi}_{1}^{0} \ell^{-}$) after an integrated luminosity of $200 \mathrm{fb}^{-1}$. The lines show the predictions for angular distributions according to supersymmetry (solid black line, eq. 2.2), phase space (dotted blue line, eq. 2.3), and universal extra dimensions (dashed red line, eq. 2.5). The error bars on the data show the statistical uncerainty on: inner error bar: SUSY signal only; intermediate error bar: inclusive SUSY with the SUSY background subtracted; outer error: inclusive SUSY with both the SUSY and the SM backgrounds subtracted. The narrow shaded band around the SUSY expectation shows how it is modified when the sparticle masses are simultaneously changed for all sparticles by $\pm 20 \mathrm{GeV}$, as described in section 4.4. Systematic uncertainties in the SUSY and SM background subtraction are not included here, but are discussed in sections 4.2 and 4.3 .

space one or the UED-like one. This means $\cos \theta_{l l}^{*}$ does indeed measure the spin of the sleptons for this point.

In fig. 6 we present the statistical separation expected for our test points (S5 and the Snowmass points) as a function of integrated luminosity. The significance indicated is shows the gaussian-equivalent significance of each of two tests:

1. A test comparing the SUSY angular distribution (eq. 2.2) to the phase space one (eq. 2.3) - demonstrating that there is sensitivity to spin in the dynamics; and separately,

2. A test comparing the SUSY angular distribution to the UED-like one (eq. 2.5) 


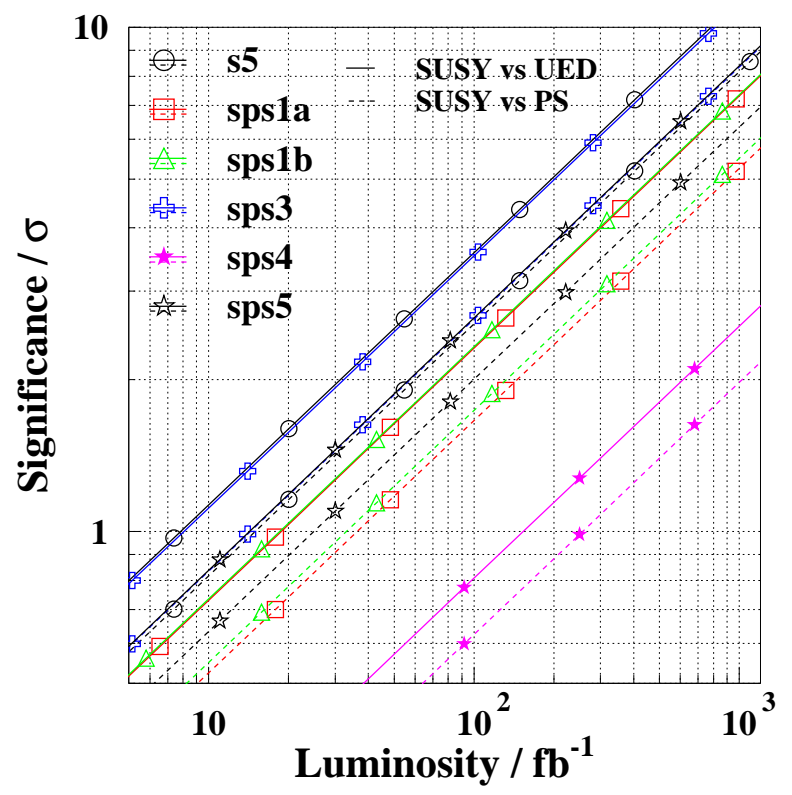

Figure 6: The expected statistical significance for discriminating SUSY from UED (solid lines), or SUSY from phase space (dashed lines) for our SUSY test points, as a function of integrated luminsity. Statistical uncertainties are included for the signal, the SUSY background from slepton-pair to gaugino (other than $\tilde{\chi}_{1}^{0}$ ) and the Standard Model backgrounds in fig. 1 . For each test point the the solid line (SUSY vs UED) always requires lower luminosity.

- showing discrimination between two physically-motivated models.

In both cases we have used a discriminant which is symmetrical in the hypotheses under test. The discriminant accounts for the fact that, because the normalisation of the distribution is fixed, the data in the bins are correlated. The events were counted in large statistics samples after the cuts had been applied and include the statistical uncertainty from the SUSY and SM background and the slepton to gaugino (other than $\tilde{\chi}_{1}^{0}$ ) background subtraction, but none of the other systematic uncertainties discussed in section 4 .

Starting with the worst case, SPS2 (from the cosmological 'focus point' region) has $>1$ TeV mass sleptons, and such a small cross-section for direct slepton production that no spin measurement (or indeed any other direct slepton production measurement) is possible for this point. It is not included in fig. 6.

SPS6 (also not on fig. 6) also presents a difficult experimental case, but for a quite different reason. This point has non-universal gaugino masses, with the $\tilde{\chi}_{1}^{0}$ more massive than would be the case with universal gauginos. Despite its large 


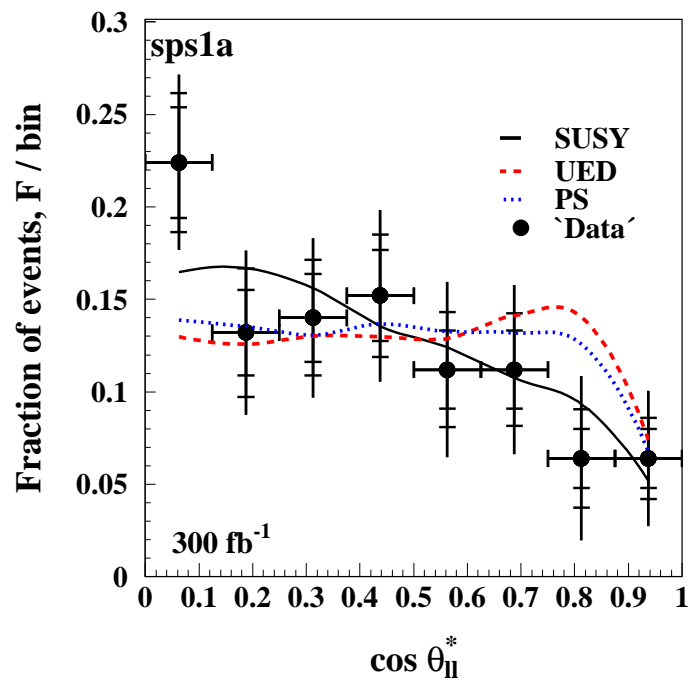

(a)

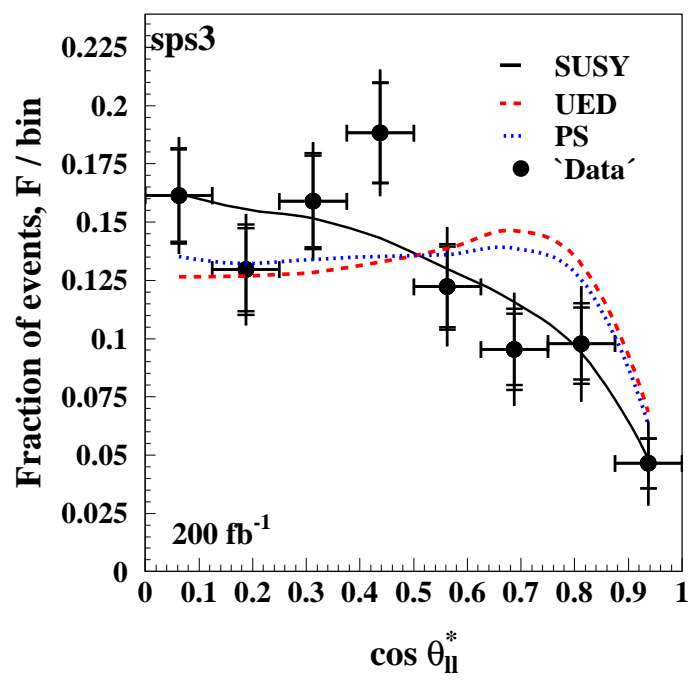

(c)

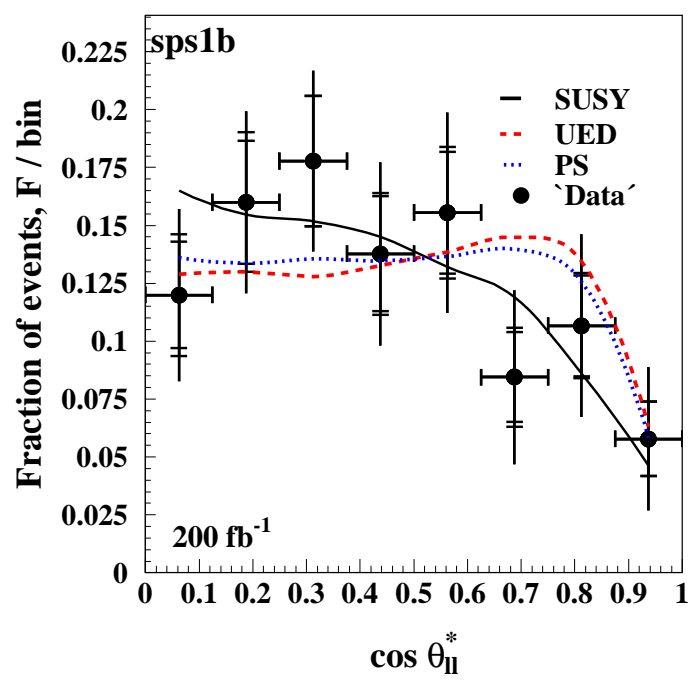

(b)

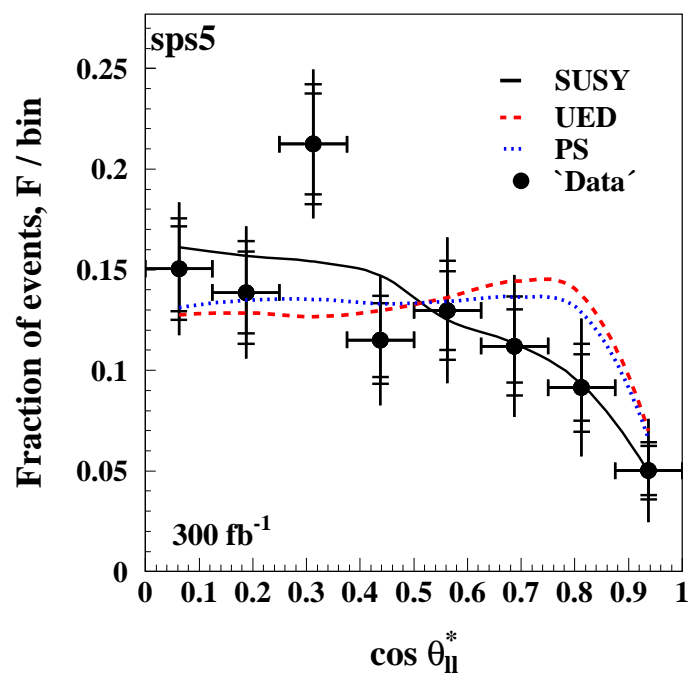

(d)

Figure 7: As for fig. 5 but for Snowmass points (a) SPS1a, (b) SPS1b (c) SPS3 and (d) SPS5. The integrated luminosity simulated in these plots is $200 \mathrm{fb}^{-1}$ for SPS1b and SPS 3, and $300 \mathrm{fb}^{-1}$ for SPS $1 \mathrm{a}$ and SPS 5 .

cross-section, a spin determination is very difficult because the sleptons are only about 50 , and $75 \mathrm{GeV}$ heavier than the LSP, and the low- $p_{T}$ leptons they produce 
end up buried beneath the $W^{ \pm} W^{\mp}$ background.

SPS4 has $\tan \beta=50$, rather heavy $\tilde{e}$ and $\tilde{\mu}$ sleptons (table 11) but relatively light $\tilde{\chi}_{1}^{+}$and $\tilde{\chi}_{2}^{0}$ at $218 \mathrm{GeV}$. The effect of this is that the signal process eq. 2.1 has to compete with cascade decays through the $\tilde{\chi}_{1}^{+}$or $\tilde{\chi}_{2}^{0}$ and so the supersymmetric background is rather large at this point. For this point, either very high statistics (such as could be achieved with an LHC luminosity upgrade [27]) or perhaps a very careful background reduction analysis, might allow the spin determination to be made in this channel.

The remaining five points, S5, SPS1a, SPS1b, SPS3, and SPS5 all allow spin determination with $100-300 \mathrm{fb}^{-1}$ of integrated luminosity. Reference distributions, similar to fig. 5, but for SPS points, are shown in fig. 7.

S5 and SPS1b which have masses 'typical' of the SUSY 'bulk' region require integrated luminosity of about 100 and $300 \mathrm{fb}^{-1}$ respectively. The point SPS5, which is distinguished by a very light stop squark, $220 \mathrm{GeV}$, behaves in this analysis like any other 'typical' spectrum. SPS1a requires a slightly higher than expected integrated luminosity (about $300 \mathrm{fb}^{-1}$ ) despite it having the largest production cross-section (table 11). This is because its lighter sleptons produce relatively soft leptons which more frequently fail the cuts, and so the cross-section after selection is actually smaller than for many of the other points.

Point SPS3 is in the cosmological 'stau co-annihilation' region, in which the lighter stau is nearly degenerate with the $\tilde{\chi}_{1}^{0}$. One might worry that the leptons it would produce might be too soft for this analysis. While this is true for the righthanded sleptons, which are only $17 \mathrm{GeV}$ heavier than the LSP, a good signal is found from the heavier left-handed sleptons, and it turns out that this point is in fact one of the easier ones with which to make this spin measurement.

In one sense fig. 6 is optimistic, in that it does not correctly take account of all the experimental systematic uncertainties explored in section 4 - only the statistical effect of the SUSY signal, and the SUSY di-slepton to gaugino and Standard Model background subtractions are included. In another sense this plot is conservative, as no tuning of the cuts has been done between the different points. With the real data, the cuts could be tuned according to the masses of the particles already measured. Such tunings have not been attempted here but have previously been shown to provide somewhat improved results for making mass measurements at different slepton masses $[14,24]$.

Overall, we can see that, unlike the previous lepton charge asymmetry measurement [5], our new method appears to be rather general. There are some difficult cases, when the sleptons are heavy or the slepton-LSP mass difference is small. However spin determination is reachable with statistics corresponding to a few years of LHC design luminosity for a variety of points coming from both the 'bulk' and the 'stau co-annihilation' regions favoured by cosmology. 


\section{Systematic uncertainties}

It is important that neither the experimental data points nor the comparison distributions are biased in a way which cannot be either measured or calculated. Having shown in section 3 that the spin measurement is within statistical reach, we now address the question of how the various systematic uncertainties can be controlled.

\subsection{Lepton efficiency, acceptance and charge identification}

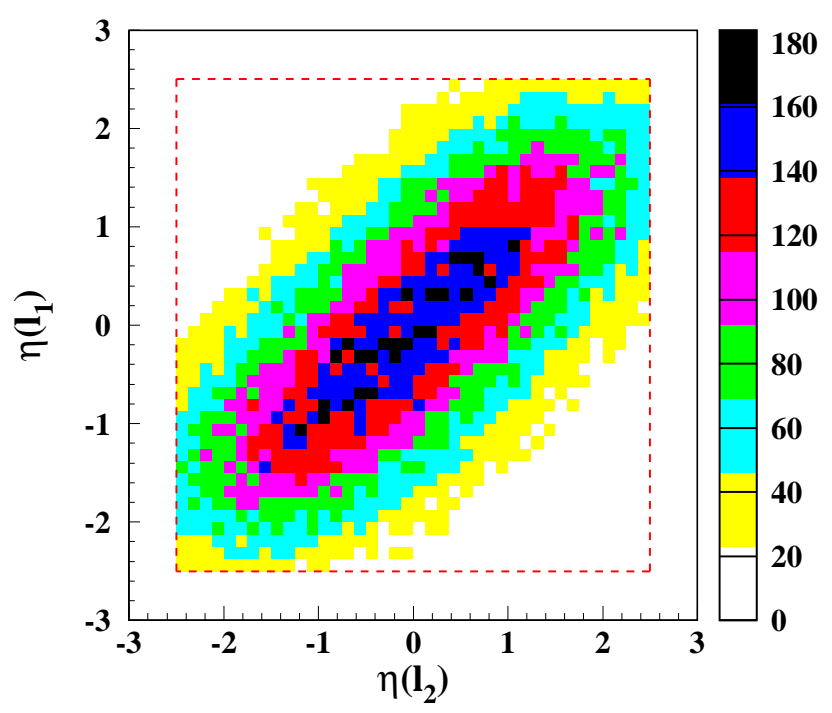

Figure 8: Reconstructed pseudorapidity $\left(\eta_{1}, \eta_{2}\right)$ of the each of the two leptons in a large sample of signal events at SUSY point S5. The ATLFAST lepton acceptance limits at $|\eta|=$ 2.5 are indicated by the dashed lines.

Since the sensitive variable is based on $\Delta \eta_{\ell^{+} \ell^{-}}$, it is clear that the electron and muon reconstruction efficiency will have to be well known across the range of pseudorapidity. In ATLAS, the reconstruction efficiency is expected to be accurately determined from the experimental data, using the high statistics sample of $Z^{0} \rightarrow \ell^{+} \ell^{-}$available at the LHC. One of the leptons is used as a tag and the efficiency is determined from the fraction of events in which the second lepton is identified. The same sample, and the same methods can be used for determining the charge mis-identification rate. This can be done for different values of $\eta$, and so the reconstruction efficiency should be well under control for all values of $\eta$, including around the barrel-endcap transition, and near the limits of the detector acceptance.

Focusing our attention more closely on the edge of the detector acceptance, we note that it is partly due loss of events beyond acceptance that there is a down-turn 


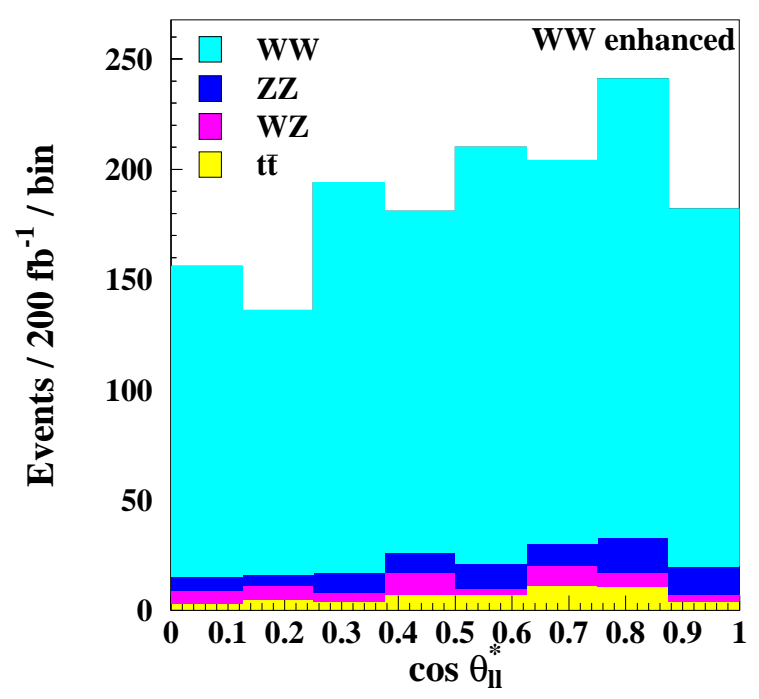

Figure 9: $\cos \theta_{l l}^{*}$ distribution for a control sample in which the $W^{ \pm} W^{\mp}$ pair contribution has been enhanced by reversing the cut on $m_{T 2}$.

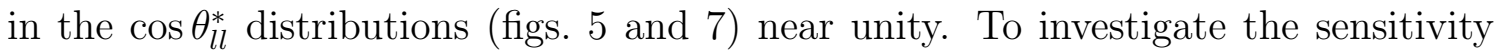
to the experimentally-forced rapidity selection we plot in fig. 8, the pseudorapidity distributions of the selected leptons. Only a small proportion of entries occur near around $(|\eta|=2.5)$, suggesting that uncertainties in the acceptance should not cause a significant bias in the results.

\subsection{Standard Model background determination}

The normalisation for each Standard Model background can be determined independantly from the data, by measuring the cross-section in regions of phase space in which each particular background dominates. For example, if one reverses the $m_{T 2}$ cut, so that only events with $m_{T 2}<100 \mathrm{GeV}$ are accepted, one obtains a sample of events rich in $W^{ \pm} W^{\mp}$ boson pairs (fig. 9). In the same spirit, it is not difficult to define cuts which preferentially select $Z^{0}$ boson pairs, $W^{ \pm} Z^{0}$ events or $t \bar{t}$ pairs. Some care obviously needs to be taken to ensure that a bias is not introduced when extrapolating from one kinematical region to another. The experimental techniques required for such an extrapolation are not investigated here, but will be the subject of a future study [28]. Previous work on SM background subtraction using the flavour subtraction methods discussed in section 4.3 has already shown good promise [14].

\subsection{SUSY backgrounds}

Although the SUSY backgrounds are generally small compared to the signal (fig. [3), 
they do need to be understood so that they can either be confirmed to be too small to worry about, or, more realistically, so that they can be accurately determined and subtracted.

Frequently-used methods $[7,24]$ for measuring the background rate from the data are based on constructing the equivalent distribution as for the signal, but rather than requiring an opposite sign, same family (OSSF) dilepton pair, asking instead for a same sign, same family (SSSF e.g. $=e^{+}, e^{+}$) pair; or an opposite sign, different family (OSDF, e.g. $\mu^{+} e^{-}$) pair; or a same sign, different family (SSDF e.g. $e^{+} \mu^{+}$) pair. Combinations of SSSF, OSDF, and SSDF selections can be employed along with particular background-enhanced samples to estimate the various backgrounds.

For high-scale processes at the LHC a flavour subtraction rather than a charge subtraction method is generally most powerful. Lepton universality is only broken by the difference between the $\mu$ and the $e$ Yukawa couplings, so distributions involving hard, isolated leptons (as opposed to those coming from near-threshold or Dalitz decays) can be generally expected to be rather universal between generations. By contrast at high scales we are sampling the proton's parton distribution functions in the valence region, so the initial state is not charge symmetric, and so neither are the final states, reducing the effectiveness of a charge-subtraction method.

For slepton pair production most of the SUSY backgrounds can be expected to have similar cross-sections in the OSSF (signal) and the OSDF (control). The good similarity between the SUSY background and an OSDF selection for our S5 point can be seen in fig. 3. It has been shown [14] that a simple OSSF-OSDF subtraction (without using control samples) can provide a rather clean background subtraction for both the SUSY background and most of the Standard Model backgrounds.

Returning for a moment to the SM backgrounds discussed in section 4.2, the size of the $W^{ \pm} W^{\mp}, W^{ \pm} Z^{0}, t \bar{t}$, and $W^{ \pm}+$'fake lepton' can also be estimated with a flavour subtraction. It is likely that the best results for both the SUSY and the SM background determinations will come from a mixture of techniques, both of OSDF subtraction and control samples.

\subsection{SUSY spectrum}

Another important issue to address is to what extent our lack of knowledge of the masses of the SUSY particles might lead to uncertainties in the comparison ("theoretical') distributions. It is safe to assume that because of the higher integrated luminosity required, any spin measurement will be made after some knowledge of the sparticles masses has been obtained. It is therefore sufficient to investigate the effect on the comparison distributions caused by varying the sparticle masses within the limits which are expected after $100 \mathrm{fb}^{-1}$ or more has been collected.

Studies $^{3}$ suggest that with around $100 \mathrm{fb}^{-1}$ of integrated luminosity the LHC

\footnotetext{
${ }^{3}$ Examples include $[11,25,29-31]$.
} 
experiments can strongly constrain the difference in masses between sparticles, but that the overall mass scale might only be known to a precision of about $10 \%$. To determine the effect the sparticle spectrum has on the SUSY comparison distribution, the masses of all of the sparticles were simultaneously raised and lowered by $20 \mathrm{GeV}$. The change in the S5 comparison distribution, indicted by the shaded band in fig. 5 was small compared to the statistical uncertainty, so we can conclude that, for this point, lack of knowledge of the SUSY mass scale will not be a significant source of systematic uncertainty. A similar test was not performed for the other points, but it can be observed (fig. (7) that the shape of the $\cos \theta_{l l}^{*}$ distributions is rather universal, despite the differences in the spectra.

\subsection{Migrations at cut boundaries}
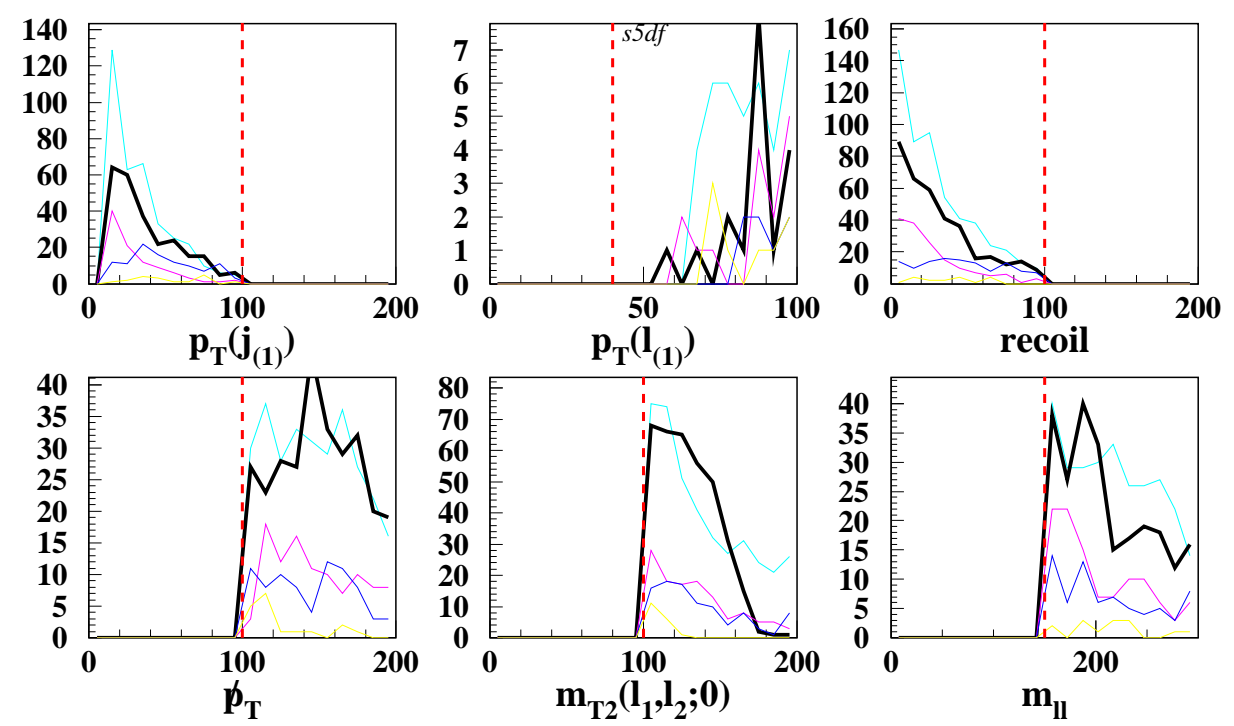

Figure 10: Distributions showing the number of events close to each of the various cuts, for signal (thick line) and backgrounds (thin lines, not to scale). The variables are the same selection as those described in section 3.1. Note that the distributions have been made after the cuts have been applied. The position of the cut is indicated by the vertical dashed line.

If the distribution of interest has a strong dependance on a poorly-known selection variable, this can generate a systematic uncertainty. Migrations might occur, for example, from uncertainty in the hadronic energy scale, or from larger jet- or missing-energy resolution due to pile-up (minimum bias) events. In fig. 10 the number of signal and SM background events (not to scale) are plotted as a funtion of the selection variables. The leading jet $p_{T}$, leading lepton $p_{T}$, and recoil have very 
few events near their corresponding cut, so migrations due to uncertainties in these variables should not significantly affect the final distributions.

The leading jet $p_{T}$ and recoil cuts are about a factor of two larger than the value $(\approx 40 \mathrm{GeV})$ at which pile-up events are expected to cause fake vetos at the percent level [11]. The number of signal events lost by jets coming from pile-up events should therefore be negligible in this analysis.

The three variables on the bottom line of fig. 10, $p_{T}, m_{T 2}$, and $m_{\ell \ell}$, do have a significant fraction of events close to their respective cuts, so a more careful study of the resolution in these variables would be appropriate. For this analysis one is particularly interested in finding out to what extent migrations might change the shape of the $\cos \theta_{l l}^{*}$ distribution.

While a detailed determination of the systematic uncertainties requires a more complete detector simulation, and is beyond the scope of this paper, we suggest that we might already have some optimism about the results. A large proportion of the visible transverse energy in the signal events is carried by the final state leptons, which should be well-measured in the electromagnetic calorimeter (electrons) or in the tracking system (muons). This means that the missing energy should also be relatively well measured, and since all three of the variables $m_{l l}, \not_{T}$, and $m_{T 2}$, depend only on $\mathbf{p}_{T \ell}$, and $\not_{T}$, they ought to be well under control.

\subsection{Migrations between bins}

Our bin size of $\Delta \cos \theta_{l l}^{*}=0.125$ corresponds to pseudorapidity differences of $\Delta \eta_{\ell^{+} \ell^{-}} \geq$ 0.25. By contrast the $\eta$ segmentation in the first sampling of the ATLAS liquid argon calorimeter in the $|\eta|<2.5$ region is in the range 0.003 to 0.006 , depending on rapidity. This fine $\eta$ segmentation will provide a much better resolution than is required for our spin measurement. Similarly, for the muon reconstruction, the rapidity measurement from the combined inner tracker and muon chambers will be much more precise than is required here - inter-bin migrations should not cause problems.

\subsection{Summary of systemic uncertainties}

In this section we have explored the various experimental systematic uncertainties which could potentially reduce the power of the $\cos \theta_{l l}^{*}$ distribution in making a spin measurement. We have found that, while there are some areas - such as in SM background estimation from data, and in understanding the effect of the missing energy resolution - which warrant further attention, there is no reason to believe there are any serious problems - no 'show-stoppers' which could invalidate the method.

\section{Conclusions}

We have described a new method for slepton spin determination at hadron colliders. 
It is based on a measurement of the di-slepton production anguar distribution, using a longitudinally boost-invariant variable we have called $\cos \theta_{l l}^{*}$.

The method is rather general, and is able to measure the spin for variety of supersymmetric model points.

We have examined LHC test point 5 (S5), and seven of the Snowmass test points (SPS1a $\rightarrow$ SPS6). Generally the results were very encouraging, though some of the test points presented particular problems. The method was unable to make a spin measurement at SPS2 (focus point region) because the production cross-section was much too small, nor at SPS6 (non-universal point) because the difference in mass between the sleptons and the lightest neutralino is small. SPS4 is a difficult case, with relatively heavy sleptons, and a large SUSY backround.

Each of the other five points tested - LHC point 5, SPS1a, SPS1b, SPS3, and SPS 5 - which include cosmological 'bulk' points, a 'stau co-annihilation' point, and a point with a light $\tilde{t}_{1}$ allowed slepton spin determination with integrated luminosity in the range 100 to $300 \mathrm{fb}^{-1}$.

There do not appear to be any systematic uncertainties which might be seriously detrimental to the measurement, but we indicate that more work does need to be done in this area.

\section{Acknowledgments}

Thank you to my colleagues in the UCL and Cambridge HEP groups, and to Shoji Asai, Giacomo Polesello, Peter Richardson and Dan Tovey for useful comments and suggestions. I have made use of the physics analysis framework and tools which are the result of ATLAS collaboration-wide efforts. This work was supported by the Particle Physics and Astronomy Research Council.

\section{References}

[1] T. Appelquist, H.-C. Cheng, and B. A. Dobrescu, Bounds on universal extra dimensions, Phys. Rev. D64 (2001) 035002, hep-ph/0012100.

[2] H.-C. Cheng, K. T. Matchev, and M. Schmaltz, Bosonic supersymmetry? Getting fooled at the LHC, Phys. Rev. D66 (2002) 056006, hep-ph/0205314.

[3] A. Datta, K. Kong, and K. T. Matchev, Discrimination of supersymmetry and universal extra dimensions at hadron colliders, hep-ph/0509246.

[4] A. Datta, G. L. Kane, and M. Toharia, Is it SUSY?, hep-ph/0510204.

[5] A. J. Barr, Determining the spin of supersymmetric particles at the LHC using lepton charge asymmetry, Phys. Lett. B596 (2004) 205-212, hep-ph/0405052. 
[6] J. M. Smillie and B. R. Webber, Distinguishing spins in supersymmetric and universal extra dimension models at the Large Hadron Collider, hep-ph/0507170.

[7] T. Goto, K. Kawagoe, and M. M. Nojiri, Study of the slepton non-universality at the CERN Large Hadron Collider, Phys. Rev. D70 (2004) 075016, hep-ph/0406317.

[8] D. Choudhury et. al., Slepton production from gauge boson fusion, Phys. Rev. D68 (2003) 075007, hep-ph/0304192].

[9] M. Battaglia, A. Datta, A. De Roeck, K. Kong, and K. T. Matchev, Contrasting supersymmetry and universal extra dimensions at the CLIC multi-TeV $e^{+} e^{-}$ collider, hep-ph/0502041.

[10] B. Bhattacherjee and A. Kundu, The International Linear Collider as a Kaluza-Klein factory, hep-ph/0508170.

[11] ATLAS Detector and Physics Peformance TDR. CERN, 1999.

[12] B. C. Allanach et. al., The Snowmass points and slopes: Benchmarks for SUSY searches, Eur. Phys. J. C25 (2002) 113-123, hep-ph/0202233.

[13] J. R. Ellis, K. A. Olive, Y. Santoso, and V. C. Spanos, Supersymmetric dark matter in light of WMAP, Phys. Lett. B565 (2003) 176-182, hep-ph/0303043.

[14] E. Lytken, Searches for direct slepton production with ATLAS, Czech. J. Phys. 54 (2004) A169-A173.

[15] G. Corcella et. al., HERWIG 6: An Event Generator for Hadron Emission Reactions with Interfering Gluons (including Supersymmetric Processes), JHEP 01 (2001) 010, hep-ph/0011363.

[16] S. Moretti, K. Odagiri, P. Richardson, M. H. Seymour, and B. R. Webber, Implementation of supersymmetric processes in the HERWIG event generator, JHEP 04 (2002) 028, hep-ph/0204123.

[17] P. Richardson, Spin correlations in Monte Carlo simulations, JHEP 11 (2001) 029, hep-ph/0110108.

[18] A. D. Martin, R. G. Roberts, W. J. Stirling, and R. S. Thorne, Parton distributions: A new global analysis, Eur. Phys. J. C4 (1998) 463-496, hep-ph/9803445.

[19] E. Richter-Was, D. Froidevaux, and L. Poggioli, ATLFAST 2.0: A fast simulation package for ATLAS, . ATLAS note ATL-PHYS-98-131.

[20] C. G. Lester and D. J. Summers, Measuring masses of semi-invisibly decaying particles pair produced at hadron colliders, Phys. Lett. B463 (1999) 99-103, http://arXiv.org/abs/hep-ph/9906349. 
[21] A. Barr, C. Lester, and P. Stephens, A variable for measuring masses at hadron colliders when missing energy is expected; $m_{T 2}$ : the truth behind the glamour, J. Phys. G29 (2003) 2343-2363, hep-ph/0304226].

[22] F. del Aguila and L. Ametller, On the detectability of sleptons at large hadron colliders, Phys. Lett. B261 (1991) 326-333.

[23] H. Baer, C.-h. Chen, F. Paige, and X. Tata, Detecting sleptons at hadron colliders and supercolliders, Phys. Rev. D49 (1994) 3283-3290, hep-ph/9311248.

[24] H. Baer, C.-h. Chen, F. Paige, and X. Tata, Signals for Minimal Supergravity at the CERN Large Hadron Collider II: Multilepton Channels, Phys. Rev. D53 (1996) 6241-6264, hep-ph/9512383.

[25] C. G. Lester, Model independent sparticle mass measurements at ATLAS. PhD thesis, 2002. CERN-THESIS-2004-003.

[26] LHC/LC Study Group Collaboration, G. Weiglein et. al., Physics interplay of the $L H C$ and the $I L C$, hep-ph/0410364.

[27] F. Gianotti et. al., Physics potential and experimental challenges of the LHC luminosity upgrade, Eur. Phys. J. C39 (2005) 293-333, hep-ph/0204087.

[28] A. J. Barr and T. Byatt., work in progress, .

[29] D. R. Tovey, Measuring the SUSY mass scale at the LHC, Phys. Lett. B498 (2001) 1-10, http://arXiv.org/abs/hep-ph/0006276.

[30] K. Kawagoe, M. M. Nojiri, and G. Polesello, A new SUSY mass reconstruction method at the CERN LHC, Phys. Rev. D71 (2005) 035008, hep-ph/0410160.

[31] C. G. Lester, M. A. Parker, and M. J. White, Determining SUSY model parameters and masses at the LHC using cross-sections, kinematic edges and other observables, hep-ph/0508143. 